DOI 10.32370/2018_09_9

\title{
STOE replaces relativity and quantum mechanics
}

\author{
J.C. Hodge ${ }^{1 *}$ \\ ${ }^{1}$ Retired, 477 Mincey Rd., Franklin, NC, 28734 \\ jchodge@frontier.com
}

\begin{abstract}
BOOk Summary: Available amazon.com, morebooks.de Paperback, 416 pages ISBN-10: 6139914655 ISBN-13: 9785139914654

The need for a paradigm shift is growing. Many ad hoc models and outlandish speculations abound to model many unexplained observations. The Scalar Theory Of Everything (STOE) audacious claim of a new paradigm is supported by corresponding to relativity and quantum mechanics; by explaining many problematical observations; and by making and fulfilling several predictions. It posits 2 components of the universe emerge to form everything. It models the universe as composed of cells with Sources (spiral galaxies) and Sinks (elliptical galaxies and matter). It forms the Universal Equations, one equation to define the potential at all points and one equation that uses the potential to exert forces on matter. These equations are applied the microwave background temperature, rotation curves, asymmetric rotation curves, galaxy redshift and discrete redshift, the pioneer anomaly, light interference, etc. The wave model of light is rejected by 2 experiments predicted by the STOE. Maxwell's Equations are modified. Life and society application of the STOE principles is suggested.
\end{abstract}

keywords: diffraction, interference, light, Afshar Experiment, STOE, TOE.

*E-mail: jchodge@frontier.com 


\section{STOE REPLACES}

relativity and quantum mechanics

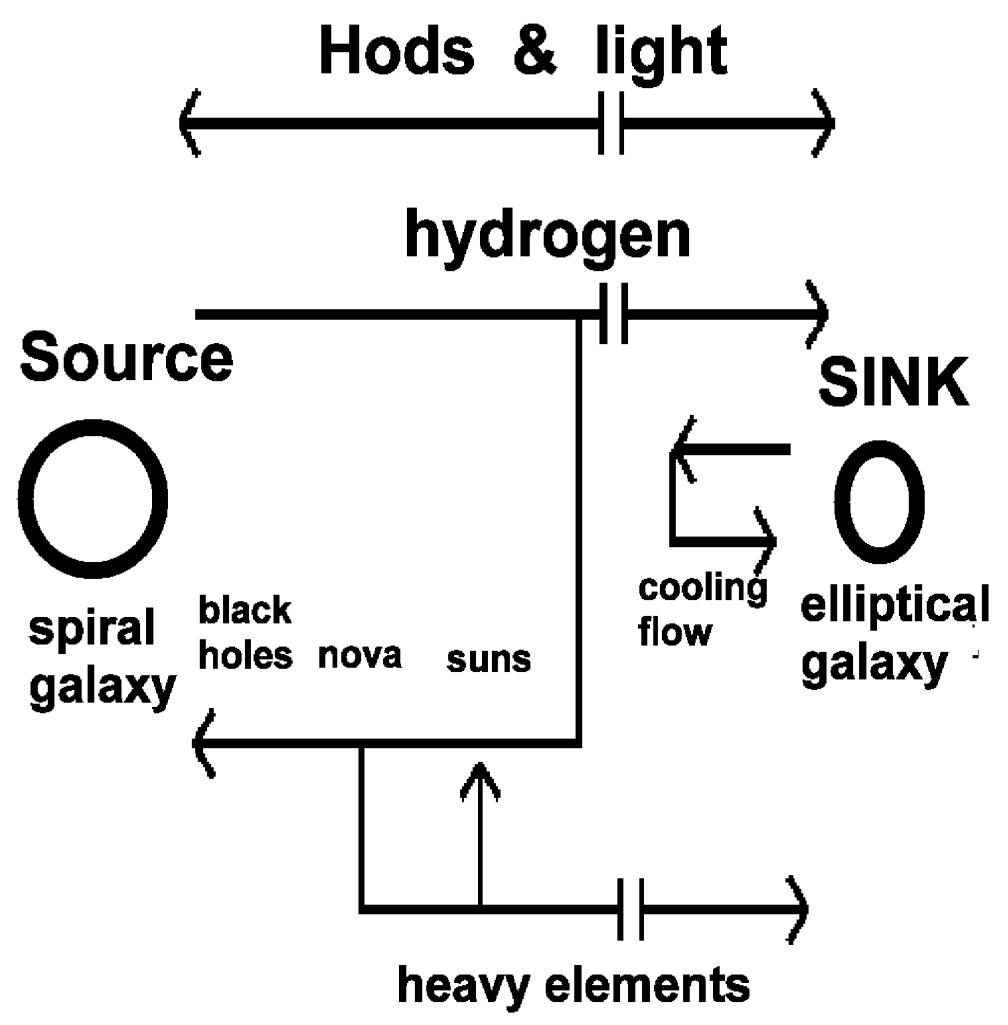

by John C. Hodge 


\section{UNIVERSAL EQUATIONS}

$$
\begin{aligned}
\rho_{\mathrm{p}}= & K_{\epsilon} \sum_{i=1}^{N_{\text {source }}} \frac{\epsilon_{i}}{r_{i}} \\
& -K_{\eta} \sum_{j=1}^{N_{\text {sink }}} \frac{\eta_{j}}{r_{j}} \\
& -K_{\text {hods }} \sum_{k=1}^{N_{\text {hods }}} \frac{K_{r}}{r_{k}} \cos \left(\frac{2 \pi r_{k}}{\lambda_{\mathrm{T}}}-\pi\right) \exp ^{-j\left(\omega t_{k}\right)} \\
> & 0 \quad K_{\mathrm{G}} \sum_{l=1}^{N_{\text {hods } l}} m_{\text {hod }}\left(\vec{n}_{l} \bullet \vec{\nabla} \rho_{\mathrm{pl}}\right) \vec{n}_{l},
\end{aligned}
$$




\section{Contents}

Acknowledgments $\quad$ xxv

$\begin{array}{ll}\text { preface } & \text { xxvii }\end{array}$

1 Postulates 1

1.1 Introduction . . . . . . . . . . . . . . . . . . . . 1

1.2 The Principles . . . . . . . . . . . . . . . . . . . . 9

2 The Universe 17

2.1 The STOE galaxy data . . . . . . . . . . . . . 54

3 GR \& QM replacement $\quad 59$

3.1 STOE . . . . . . . . . . . . . . . . . 59

3.1.1 Energy continuity equation . . . . . . . . . 66

3.1 .2 Forces . . . . . . . . . . . . . . . . 68

3.1 .3 Particles . . . . . . . . . . . . . 75

3.1.4 Chirality of matter . . . . . . . . . . . . 79

3.1.5 Source characteristics . . . . . . . . . . . 79

3.1.6 Sink characteristics . . . . . . . . . . . . . . . 79

3.1.7 Equivalence Principle . . . . . . . . . . . . . . . . . 80

3.2 Sink and Source galaxies . . . . . . . . . . . . . . . 82

3.3 Hod model . . . . . . . . . . . . . . . . . . . . . . 85

3.3.1 Hod action on $\rho$-field . . . . . . . . . . . . . . . . 88

3.3.2 $\rho$-field action on a hod . . . . . . . . . 93

3.4 Particles . . . . . . . . . . . . . . . . . . . . 94

3.5 The analogy . . . . . . . . . . . . . . . . . . . 95

3.6 Structure . . . . . . . . . . . . . . . . . . 95

3.7 spin . . . . . . . . . . . . . . . . . . . . . . 97

3.8 Universal Equations . . . . . . . . . . . . . . . . . . . 105 
4 Modify Maxwell's Equations $\quad 107$

4.1 The model . . . . . . . . . . . . . . . . . . . 107

4.2 Experiments reject the Biot-Savart Law . . . . . . . . . . . 109

4.3 Experiment 1 . . . . . . . . . . . . . . . . . . . 110

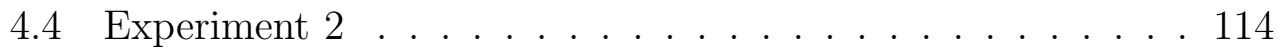

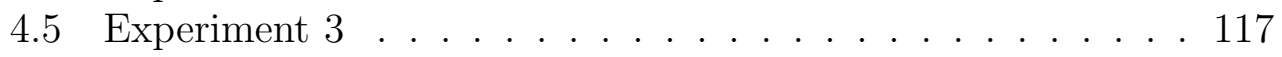

4.6 Interpretation . . . . . . . . . . . . . . . . . 117

5 Temperature of the universe $\quad 119$

5.1 Model . . . . . . . . . . . . . . . . . . . . 121

5.2 Conclusion . . . . . . . . . . . . . . . . . . . . . . 125

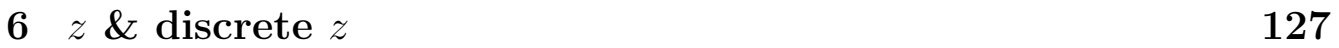

6.1 Universal Equations application . . . . . . . . . . . . . 127

6.2 Redshift model . . . . . . . . . . . . . . . . . . 130

6.3 Results . . . . . . . . . . . . . . . . . . . . 133

6.4 X factors . . . . . . . . . . . . . . . . . . . 140

6.5 Discrete variations in redshift . . . . . . . . . . . . 145

6.6 Discussion . . . . . . . . . . . . . . . . . . . . . . . . . . . 149

7 Pioneer anomaly $\quad 155$

7.1 Universal Equations . . . . . . . . . . . . . . . . . . . 155

7.2 labelpaintroIntroduction. . . . . . . . . . . . . . 155

7.3 Model . . . . . . . . . . . . . . . . . 157

7.4 Results . . . . . . . . . . . . . . . . . . 158

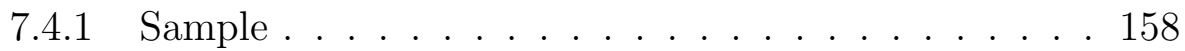

7.4.2 Annual periodicity . . . . . . . . . . . . 160

7.4.3 Difference of $a_{\mathrm{p}}$ between the spacecraft . . . . . . . . 161

7.4.4 Slow decline in $a_{\mathrm{P}} \ldots \ldots$. . . . . . . . . . . 163

7.4.5 Saturn encounter . . . . . . . . . . . . . . 163

7.4.6 Large uncertainty of P11 80/66 . . . . . . . . . 164

7.4.7 Cosmological connection . . . . . . . . . . . . 164

7.5 Discussion . . . . . . . . . . . . . . . 165

8 Rotation Velocity vs Inner Radius 169

8.1 Universal Equations . . . . . . . . . . . . . . . . . . . . . . 169

8.2 Introduction . . . . . . . . . . . . . . . . . . . . . 169

8.3 Sample . . . . . . . . . . . . . . . . . . 170

8.4 Analysis . . . . . . . . . . . . . . . . . . . . 173

8.4.1 Using DSS Data . . . . . . . . . . . . . . 175 
8.4.2 Using HST Data . . . . . . . . . . . . . . . . . . . 182

8.5 Discussion . . . . . . . . . . . . . . . . . 187

9 Distance calculation $\quad 191$

9.1 Model . . . . . . . . . . . . . . . . . . . . . . . . . 192

9.2 Data and Analysis . . . . . . . . . . . . . . . . . . . . 194

9.3 Discussion . . . . . . . . . . . . . . . . . . . 202

10 RCs \& asymmetric RCs 205

10.1 Universal Equations . . . . . . . . . . . . . . . . 205

10.2 Introduction . . . . . . . . . . . . . . . . . . . 205

10.3 Spiral galaxy model . . . . . . . . . . . . . . . . . 210

10.4 Results . . . . . . . . . . . . . . . . . . . . . 211

10.4.1 Sample. . . . . . . . . . . . . . . 211

10.4.2 First approximation . . . . . . . . . . . . . 212

10.4.3 Second approximation . . . . . . . . . . . . . . . . 225

10.5 Discussion . . . . . . . . . . . . . . . . . . . . 227

11 CMO \& $\sigma$ correlation $\quad 231$

11.1 Universal Equations . . . . . . . . . . . . . . . . . . . 231

11.2 Introduction . . . . . . . . . . . . . . . . . . 231

11.3 Sample . . . . . . . . . . . . . . . . . . 235

11.4 Results . . . . . . . . . . . . . . . . . . . 237

11.5 Discussion . . . . . . . . . . . . . . . . . . 238

12 Photon diffraction and interference $\quad 241$

12.1 Universal Equations . . . . . . . . . . . . . . . . . . . 241

12.2 INTRODUCTION . . . . . . . . . . . . . . . . . . . . 241

12.3 Model . . . . . . . . . . . . . . . . . . . . 247

12.3.1 Photon action on $\rho$-field . . . . . . . . . . . . . . 249

$12.3 .2 \rho$-field action on a photon . . . . . . . . 2 250

12.4 Simulation . . . . . . . . . . . . . . . . 250

12.5 Photons traveling a long distance . . . . . . . . . . 251

12.6 Young's experiment . . . . . . . . . . . . . . . . 258

12.7 Laser . . . . . . . . . . . . . . . . . . . . . . . . 260

12.8 Afshar experiment . . . . . . . . . . . . . . 265

12.9 Discussion . . . . . . . . . . . . . . . . . 266

12.10Single Photon diffraction and interference . . . . . . . . . . 269

12.10.1 INTRODUCTION . . . . . . . . . . . . . . 269

12.10.2 Model . . . . . . . . . . . . . . . . . 270 
12.10.3 Simulation . . . . . . . . . . . . . . 271

12.10.4Screen . . . . . . . . . . . . . . 271

12.10.5 Mask . . . . . . . . . . . . . . . 271

12.10.6 Correspondence with the Fraunhofer model . . . . . . 275

12.10.7 Discussion . . . . . . . . . . . . . 275

12.11Diffraction experiment rejects wave models of light . . . . 276

12.11.1 INTRODUCTION . . . . . . . . . . . 276

12.11.2 The experiment . . . . . . . . . . . 276

12.11.3 Edge experiments . . . . . . . . . . . . . . 279

12.11.4 Discussion . . . . . . . . . . . . . . . 281

12.11.5 Conclusion . . . . . . . . . . . . . . . . 284

12.12opaque strips \& about the Afshar Experiment . . . . . . . . 284

12.12.1 INTRODUCTION . . . . . . . . . . . . . 284

12.12.2 Experiment . . . . . . . . . . . . . . 285

12.12.3 Discussion and Conclusion . . . . . . . . . . . 293

12.13with a transparent mask rejects wave models of light . . . . 293

12.13.1 INTRODUCTION . . . . . . . . . . . . . . . 293

12.13.2 The Description of the experiment . . . . . . . 293

12.13.3 The Toy Simulation of the experiment . . . . . . . . 294

12.13.4 Discussion and Conclusion . . . . . . . . . . . 294

13 Replacement of Special Relativity 299

13.1 Universal Equations . . . . . . . . . . . . . . . . . . . 299

13.2 INTRODUCTION . . . . . . . . . . . . . . . . . . . . . 299

13.3 Null experiments . . . . . . . . . . . . . . . . . . 300

13.4 Length contraction . . . . . . . . . . . . . . 300

13.5 Doppler shift . . . . . . . . . . . . . . . . 301

13.6 Clocks . . . . . . . . . . . . . . . . . . . 302

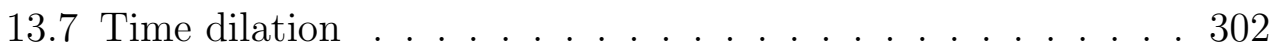

13.8 Discussion and Conclusion . . . . . . . . . . . . . . 304

$\begin{array}{ll}14 \text { Life and survival } & 305\end{array}$

14.1 The purpose of life is life . . . . . . . . . . . . . . . . 307

14.2 The nature of nature . . . . . . . . . . . . . . . . . . . . . 316

14.3 Biological to Social Mechanisms . . . . . . . . . . . . . . . 327

14.4 The Vital Way to life . . . . . . . . . . . . . . . . . . 333

15 STOE comparison to current models $\quad 347$

16 Speculations $\quad 351$ 